\title{
Embeddings on Torus-Butterfly Interconnection Network
}

\author{
Latifah \\ STMIK Jakarta STI\&K \\ Jl. BRI Radio Dalam No. 17 \\ Jakarta Selatan-Indonesia
}

\author{
Ernastuti \\ University of Gunadarma Jl. \\ Margonda Raya 100 Street \\ Depok, Indonesia
}

\author{
Djat Kerami \\ University of Indonesia \\ Depok, Indonesia
}

\begin{abstract}
This paper discuss about embedding on the new interconnection network named Torus-Butterfly. TorusButterfly is the Cartesian product network that has constant degree and has smaller network cost than the other Cartesian product network. Torus-Butterfly network is a Cayley graph. From the properties of Cayley graphs which have Hamiltonian path, the linear array and 2D-Mesh can be embedded into this new Torus-Butterfly network with minimum dilation and expansion.
\end{abstract}

\section{General Terms}

Distributed and Parallel computing systems.

\section{Keywords}

Embedded, dilation, expansion, Cayley Graph.

\section{INTRODUCTION}

Development of the network topology design is a subject of research that still continues to interest and developed. Formation model (topology) network plays an important role in parallel computing systems. The need for a lot of number of processors in the network topology is a challenge to develop various methods of designing the network topology. Several methods have been developed including the Cartesian product of two network. Some of the interconnection network that already exist was Hyper-Butterfly, which is the Cartesian product of Hypercube and the Wrap Around Butterfly [1]. The other was the Torus Embedded Hypercube which is a Cartesian product between Torus and Hypercube [2]. Another Cartesian product interconnection network was Twisted Scalable Hypercube which is the Cartesian product of linearly Scalable Hypercube and Twisted [3]. Also, the new TorusButterfly which is Cartesian product of two network topology, that is Torus and Enhanced Butterfly. The TorusButterfly interconnection networks is a Cayley graph, which a graph that widely used as the interconnection networks. This paper discuss about the embedding of linear array and 2DMesh on this new Torus-Butterfly interconnection networks.

\section{THEORITICAL REVIEW}

It is known that the structure of the model of a network can be described as a connected graph $G=(V, E)$, with $V$ the set of vertices is the set of processors and $\mathrm{E}$ is the set of edge or link in the network [4]. An edge is an ordered pair of $x, y=$ $\{x, y\}$ of distinct vertices in $G$. The set of vertices $\mathrm{V} i$ in a graph $\mathrm{G}$ is denoted as $\mathrm{V}_{\mathrm{G}}$ and the set of edge $\mathrm{E}$ in a graph $\mathrm{G}$ is denoted by $\mathrm{E}_{\mathrm{G}}$. As already mentioned in the introduction, one of the models that are widely used as interconnection networks are Cayley graphs, this is caused by the Cayley graph has the properties finite, connect, undirected and symmetry [1]. Because of the interconnection network models is considered as a graph then the term interconnection network models and a graph can be replace each other.

Definition 1: Suppose $\mathrm{H}$ is a group and $\mathrm{S} \subset \mathrm{H}$ forming a set of $\mathrm{H}$ such that $\mathrm{S}=\mathrm{S}^{-1}$. A Cayley graph of $\mathrm{H}$ against $\mathrm{S}$ is undirected graph Cay $(\mathrm{H}, \mathrm{S})$ where the set of vertices is $\mathrm{H}$ and the edge connecting g to gs for every selection $\mathrm{g} \in \mathrm{H}$ and $\mathrm{s} \in$ $\mathrm{S}$ * A Cayley graph Cay $(\mathrm{H}, \mathrm{S})$ is a regular graph $|S|$ of order $|\mathrm{H}|$ [5].

Definition 2: Suppose $d \in N$ (N = set of positive integers). Butterfly interconnection network model of dimension $\mathrm{d}$ is denoted by $\mathrm{B}(\mathrm{d})$ is a graph with vertex set $\mathrm{V}=[\mathrm{d}+1] \times[2]^{\mathrm{d}}$ and the set of edge $\mathrm{E}=\mathrm{E}_{1} \cup \mathrm{E}_{2}$ with $\mathrm{E}_{1}=\{\{(i, \alpha),(i+$ $1, \alpha)\} / i \in[d], \alpha \in[2]^{\mathrm{d}_{3}}$

and

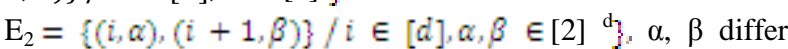
only at position $i\}$. The set of vertices $\left\{(i, \alpha) / \alpha \in[2]^{d}\right.$ is said to form the i-th level of the Butterfly [6]. Butterfly interconnection network model of dimension $\mathrm{n}$ has $\mathrm{n}$ levels and $\mathrm{N}=2^{\mathrm{n}}$ input and output. Input / output is set in the $2^{\mathrm{n}}$ columns are labeled from 0 to $2^{\mathrm{n}}-1$ (in binary). Each level is numbered $0,1,2, \ldots, \mathrm{n}$ from top to bottom [7]. The Butterfly interconnection network model only node to node in a neighboring row. Edge between the vertices in the same column is called a straight edge and the edge between nodes in different columns are called cross sections [8]. In the graph $B(d) x$ when level $d$ is replaced with level 0 , then it was said a Wrap around Butterfly dimension d or WF (d) [9]. At Wrap around Butterfly (WB) dimension $n \geq 3$ when it was added a particular edge on the graph is called Enhanced Butterfly [9].

Definition 3: Torus $(m, d)=T(m, d)$ is a graph that contains $(m, D$ mesh with wrap around sides in rows and columns [10].

Definition 4: A Mesh $(\mathrm{m}, \mathrm{l})=\mathrm{M}(\mathrm{m}, \mathrm{l})$ is a graph that consists of $m \times n$ nodes arranged in $m$ rows and $n$ columns. With the node located at the ith row and jth column is identified by an ordered pair $(\mathrm{i}, \mathrm{j}): 0 \leq \mathrm{i} \leq \mathrm{m}-1,0 \leq \mathrm{j} \leq \mathrm{n}-1[10]$.

Definition 5: Given two graphs $G=\left(\mathrm{V}_{1}, \mathrm{E}_{1}\right)_{2}$ and $H=\left(\mathrm{V}_{2}\right.$, $\left.E_{2}\right)_{2}$ Cartesian product operation defined $G$ and $H$ is denoted by $G \times H$ is the graph $T\left(V_{g} E\right)_{x}$ with $V$ and $E$ as follows 1) $V=\left\{(a, b) f a \in V_{1 D} y \in V_{2}\right\}$

2) For any $x=\left(a_{v} b\right)$ and $y=\left(c_{v} d\right)$ in $V_{v}\left(x_{v} y\right)$ is an edge in $E$ if and only if $(a, c)$ is an edge in $E_{1}$ and $b=d$ or $(b, d)$ is an edge in $E_{2}$ and $a=\mathbb{E}$ [11].

Proposition 1: Suppose that Cay $(\mathrm{S}, \mathrm{G})$ and Cay $\left(\mathrm{S}^{\prime}, \mathrm{G}^{\prime}\right)$ are two Cayley graph, then the Cartesian product Cay $(\mathrm{S}, \mathrm{G}) \times$ Cay $\left(S^{\prime}, G^{\prime}\right)=H$ is also a Cayley graph [12]. 
Definition 6: If $\mathrm{G}=\left(\mathrm{V}_{1}, \mathrm{E}_{1}\right)$ is the Torus interconnection network model of size $m l$ and $\mathrm{H}=\left(\mathrm{V}_{2}, \mathrm{E}_{2}\right)$ is the Enhanced Butterfly interconnection network model dimension $n$, then the Torus-Butterfly interconnection network model, denoted as TB $(m, l, n)$, is the Cartesian product of Torus and Enhanced Butterfly, with $m$ and $l$ is the size of Torus interconnection network model and $n$ is the dimension of the Enhanced Butterfly interconnection network model. This is true for $n \geq 3, m \geq 2$ and $l \geq 2$ [13].

Figure 3 is Torus-Butterfly network TB $(2,2,3)$.

Theorem 1: Enhanced Butterfly graph is a Cayley graph [9].

Theorem 2: Torus is a Cayley graph [14].

Definition 7: Linear array interconnection network topology LA $(\mathrm{N}), \mathrm{N} \geq 2$, is a graph that has $\mathrm{N}$ vertices consisting of two vertices of degree one, and (N-2) vertices of degree two [15].

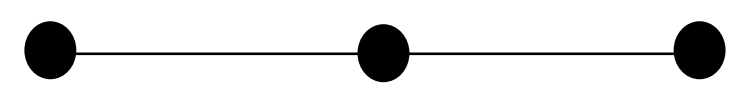

Figure 1: 3-Linear Array

Theorem .3: Cayley graph has a Hamiltonian cycle [16].

Definition 8: Embeddings means matching the logic of two interconnection network topology $\mathrm{G}$ and $\mathrm{H}$ [17].

Graph $\mathrm{G}$ is called a Guest graph and a graph $\mathrm{H}$ as a host graph.

Embeddings is very important, that is, when a programmer to solve a problem only to have algorithms that work on the interconnection network A, whereas only available interconnection network B.

Example: a linear array can be embedded into a 2 x 3 Mesh as Figure 2:

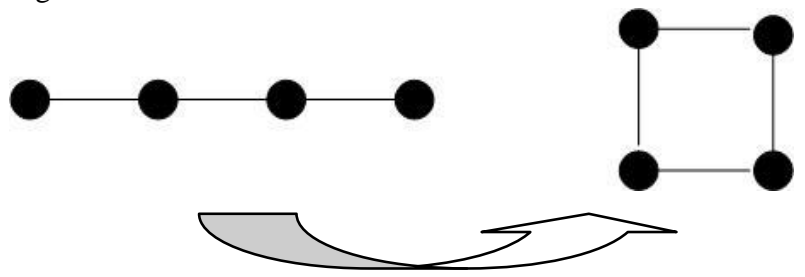

Figure 2. Embedding 4-linear array to $2 \times 2$ Mesh

At Embedding techniques in a graph by $\mathrm{G}$ and $\mathrm{H}$, respectively graph Guest and Host, required number of nodes in $\mathrm{G}$ is less than or equal to the number of nodes in $\mathrm{H}$. Efficiency emulation $\mathrm{G}$ to $\mathrm{H}$ can be measured from the parameters of dilation, expansion, congestion [15]. Ideally dilation and expansion is expected to be minimum. Here is the definition:

Definition 9: dilation of a segment $\mathrm{x}$ in a graph $\mathrm{G}$ is the length of the path in which $\mathrm{x}$ is mapped. Dilation of an investment is the maximum value of all dilation segment in $\mathrm{G}$ [18].
Definition 10: Expansion of an investment is the ratio of the number of nodes in $\mathrm{G}$ to the number of nodes in $\mathrm{H}$ [18].

In this paper it is assumed mapping between nodes and arcs of the graph Guest and Host graph is one-on-one (one-to-one).

\section{RESULTS AND DISCUSSION}

The results of this research was:

Lemma 1: Torus-Butterfly interconnection Network topology is a Cayley graph.

Proof: Torus and Enhanced Butterfly interconnection networks respectively are Cayley graphs. By proposition 1 Cartesian product between the two interconnection networks Cayley graphs is also a Cayley graph. Thus the TorusButterfly interconnection network is a Cayley graphs.

Lemma 2: Torus-Butterfly interconnection Network topology has a Hamiltonian path.

Proof: since the Torus-Butterfly interconnection network topology is Cayley graphs and each Cayley graph has a Hamiltonian path, then the Torus-Butterfly interconnection network topology has a Hamiltonian path.

Lemma 3: A linear array LA ( $\mathrm{mln} 2^{\mathrm{m}}$ ) can be embedded into $\mathrm{TB}\left(\mathrm{m}_{w} \mathbb{n}\right)$ with dilation $=1$ and expansion $=1$, for $m \geq 2, l$ $\geq 2, n \geq 2$.

Proof: The number of nodes in the TB $\left(m_{v} \mathbb{l}_{v} n\right)$ is $\left(m i n 2^{n n}\right)$. Lemma 2 says there is a Hamiltonian path in TB $\left(m_{w} \mathbb{L}_{w} n\right)_{w} m 2_{w} \mathbb{l} \geq 2 n \geq 3$ while a Hamiltonian path is a linear graph, thus proving that LA $\left(m i n 2^{\text {sh }}\right)$ can be embedded into $\mathrm{TB}\left(\mathrm{m}_{\mathrm{v}} \mathbb{R}_{\mathrm{n}}\right)$, with dilation $=1$ and the ratio (the number of nodes in LA $\left(m i n 2^{\text {nI }}\right)$ ) / (number of nodes in $\left.\mathrm{TB}\left(m_{v} \mathbb{R}_{w} x\right)\right)=\left(\min 2^{\mathrm{m}}\right) /\left(m i n 2^{\mathrm{m}}\right)=1$.

Lemma 4: A Mesh $\left(m l, n 2^{\text {sI }}\right)$ can be embedded into TB $\left(m_{v} \mathbb{R}_{v} n\right)$ with dilation $=1$ and expansion $=1$, for $m \geq 2, l \geq 2$, $n \geq 2$.

Proof: 2D Mesh (x, y) is a graph consisting of nodes arranged in rows and columns, with rows and columns are $\mathrm{x}$ and $\mathrm{y}$. Each line of the 2D Mesh can be regarded as a linear array, so the 2D Mesh can be embedded into Torus-Butterfly with dilation $=1$ and expansion $=($ number of nodes on the $2 \mathrm{D}$ Mesh) / (number of nodes on the TB) $=\frac{m \ln 2^{\mathrm{n}}}{m \ln 2^{\mathrm{n}}}=1$.

The comparative of embedding between Torus-Butterfly (TB), Hyper-Butterfly (HB) and Torus-embedded-Hypercube (TH) interconnection networks is given in Table 1. Notes that "-_" in the Table means that there were not yet any description about the embedding. 
Table 1. Comparison of embedding in three models of interconnections network

\begin{tabular}{|c|c|c|c|}
\hline Network Type & HB $(\boldsymbol{k}, \boldsymbol{n})[1]$ & TH $(16,16, k)[2]$ & TB $(\boldsymbol{m}, \boldsymbol{l ,}, \boldsymbol{n})$ \\
\hline Mesh & yes & - & Yes \\
\hline Linear Array & - & - & Yes \\
\hline
\end{tabular}

\section{CONCLUSIONS}

Linear array and 2D-Mesh can be embedded into the new Torus-Butterfly interconnection network with minimum dilation and expansion that is 1 . For future work it can be evaluated whether another interconnection network such as tree can be embedded into this new Torus-Butterfly interconnection network with minimum dilation and expansion.

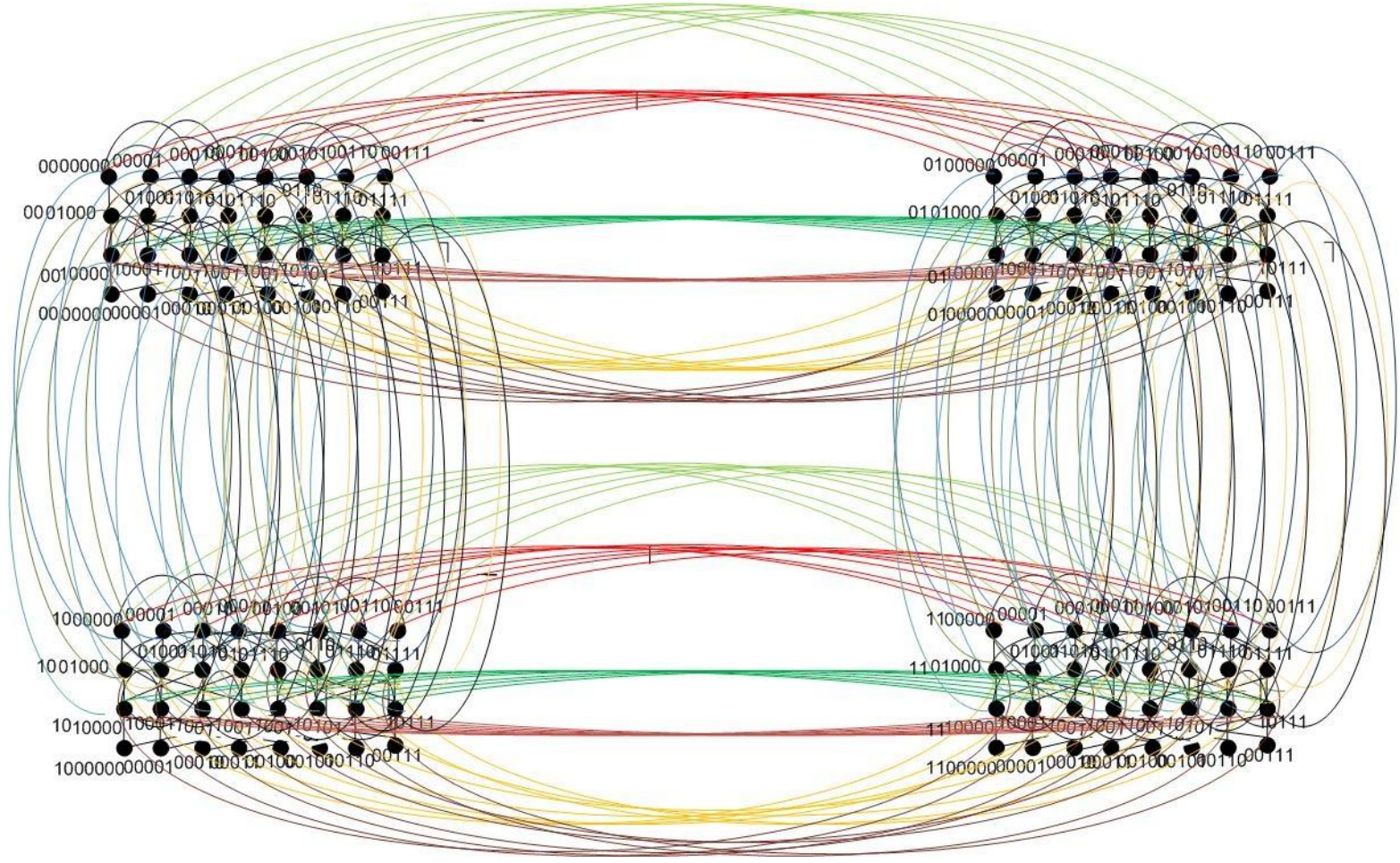

Figure 3: Torus-Butterfly Network $\operatorname{TB}(2,2,3)$

\section{ACKNOWLEDGMENTS}

Our thanks to the University of Gunadarma and STMIK Jakarta STI\&K who have sponsored this research.

\section{REFERENCES}

[1] Shi, Wei and Srimani, Pradip K, 1998. Hyper-Butterfly Network: A scalable Optimally Fault Tolerant Architecture, University of Colorado.

[2] Kini, N. Gopalakrishna, Kumar, M.Sathish, HS.Mruthyunja, 2010. Torus Embedded Hypercube Interconnection Network: A comparative Study, Journal on Computer Science and Engineering Vol 1(4), pp. 2931.

[3] Alam, Jahangir, Kumar Rajesh, 2011. STH:A Highly Scalable and Economical Topology for Massively Parallel Systems, Indian Journal of Scince Technology, Vol. 4 No. 12 pp 1737-1748.

[4] Bermont, J-C, Darrot, O, Delmas and Prennes, S, 1995, Hamilton Cycle Decomposition of The Butterfly
Network, Parallel Processing Letter, World Scientific Publishing Company pp 3-14.

[5] Liaw, sheng, chyang dan Chang, Gerard J., Wide Diameters of Butterfly Networks, Taiwanese Journal of Mathematics, Vol 3, No. 1,pp.83-88, March, 1999.

[6] Kothapalli, Kishore, 2008, Parallel Algorithms and Programming, cstar.iiit.ac.in/kkishore/cs5118/week2.pdf, pp3-6

[7] Hou, Xinmin, Xu, Jun-Ming and Xu, Min, 2009. The forwarding Indices of Wrapped Butterfly Networks, Networks,DOI 10.1002/net.

[8] Guzide, Osman dan Wagh, Meghanad D, 2006. Mapping cycles and Trees on Wrap Around Butterfly Graphs, SIAM Journal Computation, vol. 35, No. 3, pp 741-765.

[9] Guzide, Osman dan Wagh Meghanad D, 2007. Enhanced Butterfly : A Cayley Graph with Node 5 Network, ISCA International Conference on Parallel and Distributed system, view as html www.informatik.unitrier.de/ ley/db/.../ISCApdcs2007.ht $\mathrm{ml}$. 
[10] Xiang, yonghong, 2008. Interconnection Networks for Parallel and Distributed Computing, Department of Computer Sciences, University of Durham, United Kingdom.

[11] Mowshowitz, Abbe, Mitsuo, Valia and Benz, Graham, 2009, Topologist of Network Growth by Combination, City College.

[12] Day, Khaled, Al-Ayyoub, Abdel-Elah, 1997, The Cross Product Interconnection Networks, IEEE Transaction on Parallel and Distributed Systems, Vol. 8 No. 2, pp 109118

[13] Latifah, Ernastuti, Kerami, Djati, 2012, Structural Properties of Torus-Butterfly Interconnection Network, IJCA, May Edition, Vol 46(16), pp 31-35

[14] Zhang, Zhen, 2011, Some Properties in Hexagonal Torus as Cayley Graph, ICICIS, Part II, pp. 422-428
[15] Ernastuti, 2008, The New Interconnection Network Topology: Extended Lucas Cube Topology, Dissertation, Gunadarma University

[16] Mooris, Dave, 2006, Open Problems on Hamiltonian Cycles in Cayley graphs, www.people.uleth.Ca/mdavemorris

[17] Seo, Jung-Hyun, Sim, Hyun, Park, Dae Hon, Park, JangWoo and Lee, Yang Sun, 2011, One-to-one Embedding between Honeycomb Mesh and Peterson-Torus Networks, Journal On coomunication Network, Vol. 2011 pp 1959-1971

[18] Wu, J., 1997, Extended Fibonacci Cubes, IEEE Trans. On Parallel and Distributed Systems, Vol. 8 (12), pp. $1203-1210$ 